\title{
Adaptación y validación de la versión española del Sexual Cognitions Checklist (SCC)
}

\author{
Nieves Moyano* y Juan Carlos Sierra
}

Universidad de Granada (España)

\begin{abstract}
Resumen: Se presenta la adaptación y validación de la versión española del Sexual Cognitions Checklist (SCC), un autoinforme que evalúa la frecuencia con que se experimentan las fantasías sexuales, entendidas éstas como pensamientos sexuales positivos (PSP) y como pensamientos sexuales negativos (PSN). La muestra estuvo formada por 1320 individuos (593 hombres y 727 mujeres) con edades comprendidas entre 18 y 73 años; todos ellos mantenían una relación de pareja estable y actividad sexual. Para examinar la dimensionalidad del SCC, se llevó a cabo un análisis de contenido, tomando como referencia la propuesta teórica de Wilson (Fantasías Intimas, Exploratorias, Sadomasoquistas e Impersonales). De este modo, la versión inicial de 56 ítems quedó reducida a 28 ítems distribuidos en una estructura tetrafactorial que fue ratificada a través de análisis factorial confirmatorio (AFC). Asimismo, se realizó análisis de invarianza entre PSP y PSN, y por sexo. La fiabilidad osciló entre .66 en Fantasías Impersonales y .87 en Fantasias Intimas. Los PSP mostraron correlaciones positivas con actitud favorable hacia las fantasías y capacidad de ensoñación sexual. Los PSN mostraron correlaciones negativas con actitud favorable hacia las fantasías y con una dimensión de ensoñación sexual. Se discute la relevancia de distinguir los pensamientos sexuales positivos de los negativos.

Palabras clave: Sexual Cognitions Checklist; fantasías sexuales; pensamientos positivos y negativos; fiabilidad; validez.
\end{abstract}

\section{Introducción}

La mayoría de personas admite que experimenta fantasías en su actividad sexual, durante la masturbación o cuando sueñan despiertos (Zamboni y Crawford, 2002). En la actualidad se admite la importancia de las fantasías sexuales, asumiendo que su presencia puede ser un indicador de salud sexual al estimular distintos aspectos de la respuesta sexual humana (Fuertes y López 1997; Santos-Iglesias, Calvillo y Sierra, 2011; Trudel, 2002; Zimmer, Borchardt y Fischle, 1983) y estando su ausencia asociada a distintos trastornos sexuales (Nutter y Condron, 1985; Sierra, Zubeidat, Carretero-Dios y Reina, 2003). Por ello, resulta importante utilizar las fantasías como herramienta para la mejora de la vida sexual (Sierra y Buela-Casal, 2002). Asimismo, se ha demostrado que determinados tipos de fantasías podrían adquirir un carácter de disfuncionalidad sobre la salud sexual (Zubeidat, Ortega y Sierra, 2004).

Las fantasías sexuales han sido generalmente descritas como pensamientos placenteros y voluntarios (Leitenberg y Henning, 1995); sin embargo, a partir del estudio de Byers, Purdon y Clark (1998) se evidencia la existencia de dos tipos de fantasías o pensamientos sexuales: pensamientos sexuales positivos y pensamientos sexuales negativos (en lo sucesivo PSP y PSN, respectivamente), al demostrar que el 84\% de la muestra evaluada había experimentado negativamente al

* Dirección para correspondencia [Correspondence address]: Nieves Moyano. Facultad de Psicología. Universidad de Granada. Campus Universitario de la Cartuja, s/n. 18011 Granada (España).

E-mail: nmoyano@ugr.es
Title: Spanish adaptation and validation of the Sexual Cognitions Checklist (SCC)

Abstract: The Spanish adaptation and validation of the Sexual Cognitions Checklist (SCC) is here presented. This self-report measure assesses frequency of sexual fantasies, in terms of both positive sexual cognitions (PSC) and negative sexual cognitions (NSC). Sample was made up of 1.320 participants (593 males and 727 females), with ages ranging between 18 and 73 years old. All participants reported sexual activity within a longterm relationship. In order to examine the SCC dimensionality, a content analysis was carried out following Wilson's theoretical proposal (intimate, exploratory, sadomasochistic and impersonal fantasies). The original version of 56 items was reduced into 28 items, distributed across a four dimension structure, which was confirmed through Confirmatory Factor Analysis (CFA). Invariance analysis for PSP and PSN and by sex was tested. Cronbach's alpha values ranged from .66 for Impersonal Fantasies to .87 for Intimate Fantasies. PSC dimensions showed positives correlations with positive attitude towards sexual fantasies and with sexual daydreaming. Significant negative correlations were observed between NSC and positive attitude towards sexual fantasies and a dimension of sexual daydreaming. Importance of distinguishing between positive and negative sexual cognitions is discussed.

Key words: Sexual Cognitions Checklist; sexual fantasies; positive and negative thoughts; reliability; validity.

menos uno de los 20 pensamientos sexuales que les fueron presentados en una lista. Posteriormente, Renaud y Byers (2001) indicaron que, en tanto los PSN se relacionan a mayores intentos por ser controlados y son experimentados como intrusivos, los PSP están asociados a la voluntariedad. Los autores enfatizan la importancia de hacer distinguibles ambos tipos de pensamientos, ya que cada uno de ellos influiría de modo diferente sobre la conducta sexual (Renaud y Byers, 2001). Renaud y Byers (1999, p. 20) definen los PSP como "pensamientos experimentados como aceptables, placenteros y egosintónicos", en tanto que los PSN constituirían "pensamientos inaceptables, desagradables y egodistónicos". Se debe señalar que los pensamientos sexuales pueden ser positivos y negativos de forma simultánea y que los componentes que los definen (aceptable/inaceptable, placentero/no placentero, egosintónico/egodistónico) no son mutuamente excluyentes (Renaud y Byers, 2001).

Teniendo en cuenta la relevancia de las fantasías sexuales en la salud sexual, es necesario disponer de instrumentos que permitan evaluar su frecuencia, permitiendo distinguir entre PSP y PSN. El Sexual Cognitions Checklist (SCC; Renaud y Byers, 1999; Renaud y Byers, 2011) es un instrumento que permite esta evaluación. Cuarenta de los ítems que lo forman fueron tomados del Wilson Sex Fantasy Questionnaire (WSFQ; Wilson, 1988) y los 16 restantes fueron extraídos del Revised Obsessive Intrusives Inventory, Sex Version (ROII-v2; Purdon y Clark, 1994). De este último instrumento se tomó además la escala de respuesta. Así, cada uno de los 56 ítems que componen el SCC son respondidos en dos escalas de respuesta tipo Likert de 7 puntos, que van desde 0 (nunca be tenido este pensamiento) hasta 6 (tengo este pensamiento de forma fre- 
cuente durante el día), permitiendo evaluar cada pensamiento sexual como positivo y negativo. Sus autores informan de una consistencia interna para la escala positiva de .95 en hombres y mujeres, y para la escala negativa de .96 en hombres y .95 en mujeres. Respecto a su validez, se ha demostrado que los PSP se relacionan con mayores niveles de afecto positivo, excitación sexual, frecuencia masturbatoria, número de parejas, satisfacción y mejor ajuste sexual, en tanto que los PSN no están asociados a un peor ajuste sexual (Renaud y Byers, 2001).

El objetivo de este estudio instrumental (Carretero-Dios y Pérez, 2007; Montero y León, 2007) es adaptar y validar el Sexual Cognitions Checklist en población española. Como indicadores de la validez de sus medidas se correlacionarán sus puntuaciones con las del Hurlbert Index of Sexual Fantasy (Hurlbert y Apt, 1993) y el Sexual Daydreaming Scale (Giambra y Singer, 1998). En este sentido, se espera encontrar una relación positiva de los PSP con la actitud favorable hacia las fantasías sexuales (Desvarieux, Salamanca, Ortega y Sierra, 2005; Sierra, Ortega y Zubeidat, 2006) y la capacidad de ensoñación sexual (Viedma, Gutiérrez, Ortega y Sierra, 2005) y no existirá relación o ésta será negativa entre PSN y actitud favorable hacia las fantasías y capacidad de ensoñación sexual (Byers et al., 1998; Purifoy, Grodsky y Giambra, 1992).

\section{Método}

\section{Participantes}

La muestra, seleccionada de forma incidental, está compuesta por 1320 sujetos ( $44.9 \%$ hombres y $55.1 \%$ mujeres) con un rango de edad comprendido entre 18 y 73 años $(M=$ 31.84; $D T=12.30)$, siendo la media de edad en hombres $34.59(D T=13.38)$ y en mujeres $29.60(D T=10.86)$. En cuanto al nivel educativo, un $6.9 \%$ informó tener un nivel de estudios de primaria, un $18.6 \%$ estudios secundarios y un $74.2 \%$ estudios superiores. Todos los participantes mantenían una relación de pareja heterosexual desde hacía al menos seis meses, con actividad sexual dentro de la misma. La muestra fue recabada en las comunidades autónomas de Andalucía y Madrid.

\section{Instrumentos}

Sexual Cognitions Checklist (SCC; Renaud y Byers, 1999, Renaud y Byers, 2011). La versión original está formada por 56 ítems contestados en dos escalas de respuesta que evalúan la frecuencia con la que se experimenta cada pensamiento sexual de forma positiva y de forma negativa. Las propiedades psicométricas han sido anteriormente comentadas.

Versión española del Hurlbert Index of Sexual Fantasy (HISF; Hurlbert y Apt, 1993) de Desvarieux et al. (2005). Permite evaluar la actitud favorable hacia las fantasías sexuales a través de 10 ítems contestados en una escala tipo Likert de 5 puntos desde 0 (nunca) a 4 (siempre). Presenta una consistencia interna igual a .85. La actitud hacia las fantasías sexuales mostró correlaciones con diferentes tipos de fantasías sexuales, deseo sexual (Desvarieux et al., 2005) y capacidad de ensoñación sexual (Perla, Sierra, Vallejo-Medina y Gutiérrez-Quintanilla, 2009). $\mathrm{El}$ alfa de Cronbach alcanzado en este estudio fue igual a 90 .

Versión española del Sexual Daydreaming Scale (SDS; Giambra y Singer, 1998) de Viedma et al. (2005). Sus 11 ítems contestados en una escala tipo Likert de 5 puntos desde 0 (totalmente falso en mi) a 4 (muy cierto en mi) permiten evaluar la capacidad de ensoñación sexual y se agrupan en tres subescalas (Tendencia a ensoñaciones heterosexuales, Intensidad de ensoñaciones sexuales y Ensoñaciones eróticas personalizadas) con coeficientes de fiabilidad iguales a .82, .74 y .71 , respectivamente. Sus puntuaciones correlacionaron de forma significativa con la frecuencia de distintos tipos de fantasías sexuales y con el deseo sexual (Viedma et al., 2005). El alfa de Cronbach obtenido en este estudio fue $.84, .68$ y .71 respectivamente, para las tres subescalas.

\section{Procedimiento}

En primer lugar se realizó la traducción de los ítems del SCC al castellano por un psicólogo bilingüe y un experto en temática sexual de modo conjunto, utilizando el procedimiento de traducción directa o hacia delante (Hambleton, 1993, 1996). La traducción realizada fue evaluada de modo individual por otros dos expertos en temática sexual y discutida conjuntamente, considerándose la redacción y comprensión de los ítems. Se realizaron modificaciones en los ítems 4, 47 y 49, aunque ninguna supuso un cambio sustancial en el contenido del ítem, sino que estuvieron dirigidas a evitar la literalidad de la traducción y mejora de su comprensión en castellano. Posteriormente, y siguiendo la propuesta de Hambleton y Patsula (1999), se realizó un estudio piloto en el que 25 sujetos con características similares a los que conformarían la muestra final indicaron si comprendían correctamente cada uno de los ítems, señalando los términos que resultaban ambiguos y sugiriendo, si fuese el caso, un enunciado alternativo. Los ítems 18 y 47 no alcanzaron un $85 \%$ de acuerdo sobre la claridad de alguno de sus términos, por lo que se añadió una breve aclaración o ejemplo entre paréntesis en esos ítems.

Tras la elaboración de la versión definitiva en castellano del SCC, un único evaluador se encargó de publicitar el estudio a través de un cartel informativo en la Biblioteca de Andalucía en Granada, contactar con diversos Centros de Formación de Adultos, Ciclos Formativos y Talleres de Empleo de Andalucía, así como solicitar la colaboración en el estudio en diversas clases de la Facultad de Psicología de la Universidad Complutense de Madrid, siendo los instrumentos administrados en un aula de cada uno de los citados lugares en pequeños grupos, los cuales no excedieron de 30 
sujetos, o haciendo entrega de la batería de instrumentos de modo individual y siendo recogidos en días posteriores. Todos los participantes recibieron las mismas instrucciones, se les informó de los objetivos del estudio y de la voluntariedad para colaborar en el mismo. Su anonimato y confidencialidad fueron garantizados a través de un consentimiento informado. El tiempo aproximado en cumplimentar la batería de instrumentos fue de 45 minutos.

\section{Resultados}

Previo al análisis de la dimensionalidad del instrumento, se consideraron las siguientes cuestiones: a) la construcción original del SCC está basada en la combinación de ítems pertenecientes a dos instrumentos independientes; b) cada uno de los ítems es contestado en dos escalas de respuesta (PSP y PSN); y c) no existe ningún estudio previo que haya analizado la estructura factorial del SCC. Por ello, se llevaron a cabo los siguientes análisis: a) análisis de contenido tomando como referencia el planteamiento teórico de Wilson de cuatro tipos de fantasías sexuales (intimas, exploratorias, sadomasoquistas e impersonales); b) análisis factorial confirmatorio (AFC) sobre las puntuaciones obtenidas en las dos escalas de respuesta correspondientes a PSP y PSN; y c) análisis de invarianza entre PSP y PSN, y por sexo.

\section{Análisis de contenido}

El juicio fue llevado a cabo por ocho expertos, todos ellos especialistas en el campo de la sexualidad humana. Cada experto debía: 1) indicar la pertinencia de los ítems, valorando si su contenido es o no congruente al constructo de pensamientos sexuales, en base a la definición propuesta por Renaud y Byers (1999), y 2) especificar en qué dimensión se categorizaría cada ítem, siguiendo la definición planteada por Sierra et al. (2006) y Wilson (1988). Así, las fantasías íntimas son pensamientos relacionados con la búsqueda del placer y disfrute mediante el compromiso profundo con un número limitado de parejas sexuales; las fantasías exploratorias están referidas a la tendencia hacia la excitación a través de la variedad sexual; las fantasías sadomasoquistas aluden a la resistencia o provocación de dolor durante la excitación sexual; y, por último, las fantasías impersonales se relacionan con el interés por fetiches, ropa, películas y otras manifestaciones sexuales indirectas, dando escaso valor a los sentimientos. Posteriormente se evaluó el porcentaje de acuerdo obtenido por los expertos en cada uno de los ítems sobre su pertinencia con el constructo de pensamientos sexuales y relevancia en cada una de las dimensiones, teniendo en cuenta como niveles aceptables de acuerdo valores entre .70 (Davis, 1992) y .80 (Selby-Harrington, Mehta, Jutsum, Riportella-Muller y Quade, 1994). Los ítems que no alcanzaron en alguna de las dimensiones un acuerdo de al menos un $75 \%$, o en su defecto de al menos seis jueces, fueron eliminados (ítems 4: Tener sexo con un extraño; 7: Tener relaciones sexuales con alguien que es "tabú"; 13: Abalanzarme y besar a una figura de autoridad; 14: Subir mi falda o bajar mis pantalones, exbibiéndome en público; 19: Ser intimidado/ a por las propuestas sexuales de un extraño; 26: Participar en una relación sexual que viola mis principios religiosos; 28: Hacer el amor en otro lugar distinto al dormitorio; 30: Lastimar o dañar a la pareja; 31: Ser lastimado/a o dañado/a por la pareja; 33: Sentirse excitado/a al ver a alguien orinar, 34: Ser atado/a; 35: Masturbarme en un lugar público; 36: Figuras de autoridad desnudas; 37: Tomar parte en relaciones sexuales con personas desnudas; 39: Atar a alguien; 40: Tener relaciones sexuales incestuosas; 41: Exhibirme de forma provocativa; 44: Tener sexo con alguien mucho más joven que yo; 45: Tener sexo con alguien mucho mayor que yo; 46: Ser muy deseado/ a por el sexo opuesto; 47: Ser seducido/ a como un "inocente"; 48: Seducir a alguien "inocente"; 49: Sentirme avergonzado/a por un fracaso en la actividad sexual; 51: Usar objetos para la estimulación; 55: Decir algo a la pareja durante una relación sexual, que sé que le disgustará; y 56: Hacer algo a la pareja durante una relación sexual, que sé que le disgustarâ). Del mismo modo, los ítems que, habiendo obtenido un grado de acuerdo óptimo, no fueron agrupados en la dimensión propuesta por Wilson (1988), fueron también excluidos (ítems 3: Practicar un coito con alguien conocido, pero con quien no be tenido relaciones sexuales; y 42: Llevar prendas de vestir del sexo opuesto). De este modo, el SCC quedó formado por 28 ítems distribuidos en cuatro dimensiones equivalentes a las planteadas por Wilson (1988) (véase Apéndice).

\section{Análisis de ítems y fiabilidad}

Como se observa en la Tabla 1 , la respuesta media a los ítems de PSP osciló entre 0.34 (ítem 20) y 4.59 (ítem 2), situándose las desviaciones típicas entre 0.82 (ítem 18) y 1.71 (ítem 29). Respecto a los PSN, las medias oscilaron entre 0.26 (ítem 29) y 0.90 (ítem 6) y las desviaciones típicas entre 0.78 (ítem 1) y 1.35 (ítem 6). No se encontraron valores del índice de correlación ítem-total corregida inferiores a .30 en ninguno de los ítems, ni en la escala positiva ni en la negativa (Nunnally y Bernstein, 1995). En la Tabla 2 se recoge la información correspondiente a los estadísticos descriptivos de cada dimensión y análisis de fiabilidad para PSP y PSN. En cuanto a los PSP, la media de cada subescala osciló entre 0.86 (DT $=0.49)$ en Fantasias Sadomasoquistas y 3.75 (DT = 0.57) en Fantasías Intimas. Respecto a los PSN, las medias oscilaron entre $0.40(D T=0.08)$ en Fantasías Intimas y 0.57 (DT $=0.22$ ) en Fantasias Sadomasoquistas. Se observa que un mayor número de ítems correspondientes a PSP obtienen valores en sus medias próximos al centro del rango de respuesta de la escala, que es 3; en concreto, los correspondientes a la dimensión Intimas, en tanto que ninguno de los ítems de PSN obtienen medias superiores a 1, mostrando una escasa variabilidad en las respuestas en este tipo de fantasías. En el análisis de la consistencia interna, se obtuvieron valores alfa de Cronbach que oscilaron entre .66 en Fantasías Impersonales (PSN) y .87 en Fantasías Intimas (PSP). La fiabilidad alcanzada es similar entre cada una de las dimensiones de PSP y la correspondiente dimensión en PSN. 
Tabla 1. Análisis de ítems por dimensión en PSP y PSN del SCC.

\begin{tabular}{|c|c|c|c|c|c|c|c|c|}
\hline \multirow{3}{*}{$\begin{array}{l}\text { Fantasías Intimas } \\
\text { Ítem }\end{array}$} & \multicolumn{4}{|c|}{ PSP } & \multicolumn{4}{|c|}{$P S N$} \\
\hline & & & & & & & & \\
\hline & $M$ & $D T$ & $r_{i-t}$ & $\alpha_{-i}$ & $M$ & $D T$ & $r_{i-t}$ & $\alpha_{-i}$ \\
\hline 1.Hacer el amor al aire libre en un lugar romántico & 2.70 & 1.48 & .42 & .88 & 0.30 & 0.78 & .34 & .87 \\
\hline 2. Practicar un coito con una pareja amada & 4.59 & 1.30 & .51 & .87 & 0.34 & 0.96 & .42 & .86 \\
\hline 15. Recibir sexo oral & 3.74 & 1.57 & .69 & .85 & 0.45 & 1.07 & .67 & .84 \\
\hline 16. Proporcionar sexo oral & 3.48 & 1.65 & .67 & .86 & 0.51 & 1.11 & .58 & .85 \\
\hline 21. Recibir o dar estimulación genital & 3.63 & 1.55 & .65 & .86 & 0.52 & 1.17 & .63 & .85 \\
\hline 24. Desnudar a alguien & 3.83 & 1.54 & .71 & .85 & 0.41 & 1.09 & .68 & .84 \\
\hline 25. Ser desnudado/a & 3.65 & 1.62 & .70 & .85 & 0.43 & 1.10 & .70 & .84 \\
\hline 52. Ser masturbado/a hasta el orgasmo por la pareja & 3.62 & 1.57 & .67 & .86 & 0.31 & 0.93 & .65 & .84 \\
\hline 54. Besar apasionadamente & 4.56 & 1.35 & .55 & .87 & 0.33 & 1.06 & .66 & .84 \\
\hline \multicolumn{9}{|l|}{ Fantasias Exploratorias } \\
\hline Ittem & $M$ & $D T$ & $r_{i-t}$ & $\alpha_{-i}$ & $M$ & $D T$ & $r_{i-t}$ & $\alpha_{-i}$ \\
\hline 5. Participar en un acto sexual con alguien que tiene autoridad sobre mí & 1.10 & 1.50 & .56 & .84 & 0.49 & 0.97 & .54 & .79 \\
\hline 8. Tener sexo con dos personas a la vez & 1.83 & 1.52 & .73 & .81 & 0.45 & 0.88 & .59 & .78 \\
\hline 9. Participar en una orgía & 1.39 & 1.44 & 69 & .82 & 0.49 & 0.91 & .64 & .77 \\
\hline 12. Participar en una actividad sexual contraria a mi orientación sexual & 0.69 & 1.15 & .39 & .85 & 0.54 & 1.08 & .45 & .80 \\
\hline 32. Intercambio de parejas & 1.03 & 1.28 & .57 & .84 & 0.53 & 0.97 & .58 & .78 \\
\hline 38. Tener sexo en un lugar público & 1.75 & 1.55 & .53 & .84 & 0.37 & 0.81 & .52 & .79 \\
\hline 43. Ser promiscuo/a & 1.51 & 1.57 & .60 & .83 & 0.56 & 1.04 & .50 & .79 \\
\hline 50. Tener sexo con alguien de diferente raza & 1.55 & 1.53 & .64 & .82 & 0.27 & 0.79 & .42 & .80 \\
\hline \multicolumn{9}{|l|}{ Fantasías Sadomasoquistas } \\
\hline Item & $M$ & DT & $r_{i-t}$ & $\alpha_{-i}$ & $M$ & DT & $r_{i-t}$ & $\alpha_{-i}$ \\
\hline 6. Ser presionado/a a mantener relaciones sexuales & 0.60 & 1.21 & .63 & .77 & 0.90 & 1.35 & .57 & .83 \\
\hline 10. Ser forzado/a a hacer algo sexual & 0.62 & 1.13 & .67 & .76 & 0.74 & 1.15 & .73 & .81 \\
\hline 11. Forzar a alguien a hacer algo sexual & 0.62 & 1.12 & .59 & .78 & 0.51 & 1.12 & .72 & .81 \\
\hline 20. Ser una víctima sexual & 0.34 & 0.94 & .53 & .79 & 0.76 & 1.28 & .57 & .83 \\
\hline 22. Azotar o golpear en el trasero a alguien & 1.64 & 1.66 & .52 & .80 & 0.32 & 0.86 & .43 & .85 \\
\hline 23. Ser azotado/a o golpeado/a en el trasero & 1.27 & 1.62 & .55 & .79 & 0.34 & 1.00 & .64 & .82 \\
\hline 27. Forzar a otra persona a mantener relaciones sexuales conmigo & 0.45 & 0.91 & .45 & .80 & 0.41 & 1.06 & .62 & .82 \\
\hline \multicolumn{9}{|l|}{ Fantasias Impersonales } \\
\hline Ítem & $M$ & $D T$ & $r_{i-t}$ & $\alpha_{-i}$ & $M$ & $D T$ & $r_{i-t}$ & $\alpha_{-i}$ \\
\hline 17. Observar el acto sexual de otras personas & 1.56 & 1.49 & .52 & .56 & 0.59 & 1.08 & .54 & .52 \\
\hline 18. Tener sexo con un animal u objeto inanimado (p.e.. muñeco/a hinchable) & 0.36 & 0.82 & .34 & .68 & 0.38 & 1.05 & .33 & .67 \\
\hline 29. Ser excitado/a por material o ropa (p.e.. látex. cuero. ropa interior) & 1.89 & 1.71 & .42 & .64 & 0.26 & 0.86 & .43 & .61 \\
\hline 53. Contemplar imágenes o películas de contenido erótico & 2.45 & 1.64 & .60 & .50 & 0.44 & 1.02 & .49 & .56 \\
\hline
\end{tabular}

Nota.- M: Media, DT: Desviación típica, $r_{\mathrm{i}-\mathrm{t}}$ Correlación ítem-total corregida, $a_{-i:}$ Alfa de Cronbach si el ítem es eliminado.

Tabla 2. Media, desviación típica y alfa de Cronbach para cada dimensión en PSP y PSN.

\begin{tabular}{lrrrrrr}
\hline & \multicolumn{3}{c}{ PSP } & & \multicolumn{3}{c}{ PSN } \\
\hline & $M$ & $D T$ & $\alpha$ & $M$ & $D T$ & $\alpha$ \\
\hline Fantasías Intimas & 3.75 & 0.57 & .87 & 0.40 & 0.08 & .86 \\
Fantasias Exploratorias & 1.34 & 0.43 & .85 & 0.46 & 0.09 & .81 \\
Fantasías Sadomasoquistas & 0.86 & 0.49 & .81 & 0.57 & 0.22 & .85 \\
Fantasias Impersonales & 1.87 & 0.87 & .67 & 0.42 & 0.13 & .66 \\
\hline
\end{tabular}

\section{Análisis factorial confirmatorio}

Se realizó Análisis Factorial Confirmatorio (AFC) con AMOS 7.0 con las puntuaciones obtenidas en las dos escalas de respuesta correspondientes a PSP y PSN. Se pusieron a prueba dos modelos: a) Modelo 1, de cuatro dimensiones que fue obtenido en el análisis de contenido y b) Modelo 2, unidimensional, similar al planteamiento de Renaud y Byers (1999), quienes no hacen distinción entre los ítems según el contenido. El método utilizado fue mínimos cuadrados generalizados (GLS), dado el incumplimiento de normalidad de los datos a través de Kolmogorov-Smirnov ( $p<.001)$, siendo por tanto este método más robusto y adecuado considerándose el tamaño muestral. Dado que el valor de $\chi^{2}$ es muy sensible al tamaño de la muestra (Jöreskog y Sörbom, 1993), se tomaron como referencia los siguientes índices de ajuste: el ratio $\chi^{2} / g l$, GFI, AGFI y RMSEA. Para el ratio $\chi^{2} / g l$, valores inferiores a 3 indican un buen ajuste (Chau, 1997; Hartwick y Barki, 1994), siendo aceptables valores inferiores a 5 (Bentler, 1989). Valores mayores o iguales a .85 en GFI y AGFI son igualmente indicadores de ajuste adecuado (Jöreskog y Sörbom, 1993) y para RMSEA, un valor inferior a .05 indicaría un modelo con buen ajuste (Browne y Cudeck, 1993; Schumacker y Lomax, 2004), aceptándose valores de hasta a .06 como ajuste aceptable del modelo (Hu y Bentler, 1999). En la Tabla 3 se muestran los índices de ajuste obtenidos en cada modelo. 
Tabla 3. Índices de bondad de ajuste de los modelos para PSP y PSN.

\begin{tabular}{lccccc}
\hline & $\chi^{2}$ & $\mathrm{gl}$ & $\chi^{2} / \mathrm{gl}$ & $\mathrm{RMSEA}$ & GFI \\
\hline Pensamientos sexuales positivos & & & & & \\
\hline Modelo 1: Cuatro dimensiones & 2130.90 & 344 & 6.19 & .06 & .86 \\
Modelo 2: Unidimensional & 2633.38 & 350 & 7.52 & .07 & .88 \\
\hline Pensamientos sexuales negativos & & & & & .83 \\
\hline Modelo 1: Cuatro dimensiones & 2503.36 & 344 & 7.27 & .06 & .86 \\
Modelo 2: Unidimensional & 2831.78 & 350 & 8.09 & .07 & .84 \\
\hline
\end{tabular}

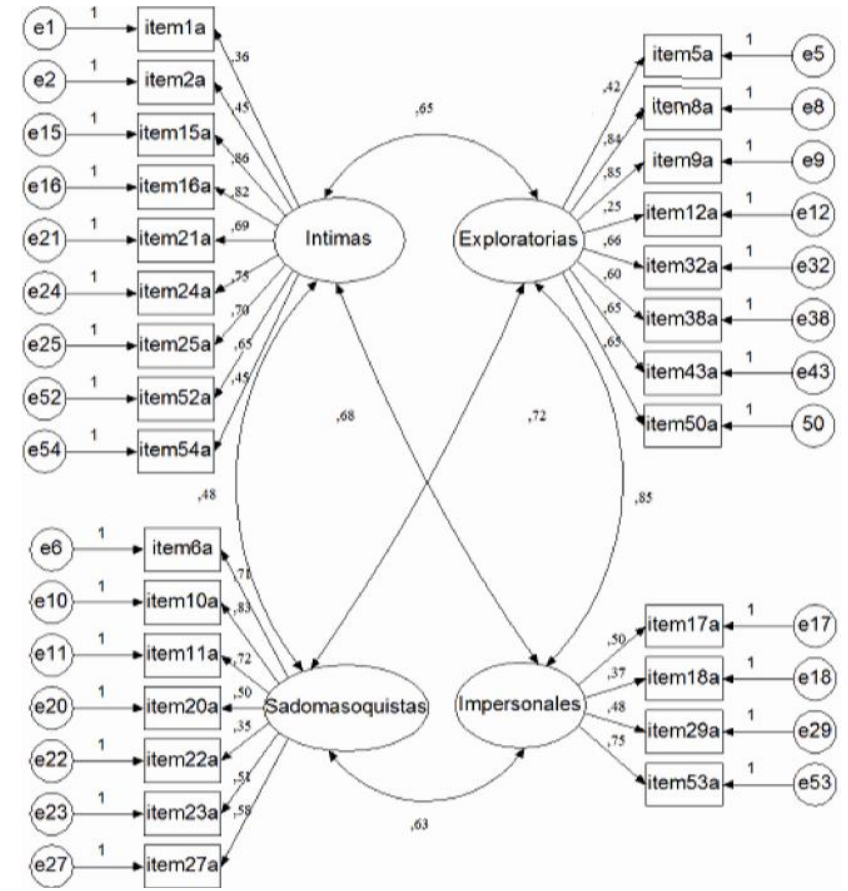

Figura 1. Path diagram del modelo de cuatro factores correspondiente a PSP del SCC-28.

El índice $\chi^{2} / g l$ no está dentro de los límites considerados aceptables en ninguno de los modelos propuestos, por lo que se consideraron los valores obtenidos en los demás índices de ajuste. Así, en los dos modelos planteados para los PSP, se observa que los índices del modelo de cuatro dimensiones son aceptables, al ser el valor de RMSEA igual a .06 y presentar GFI y AGFI valores superiores a .85. El modelo tetrafactorial muestra, por tanto, un mejor ajuste en comparación con un modelo unidimensional, al presentar este modelo valores RMSEA igual a .07, GFI igual a .85 y AGFI igual a .83. En cuanto al modelo tetrafactorial para los PSN, se encuentra que RMSEA obtiene un valor de .06, y GFI y AGFI obtienen valores de .86 y .84 respectivamente, siendo por tanto su aceptabilidad débil por no alcanzar consenso entre los índices evaluados; aunque en comparación con un modelo unidimensional, el primero resulta de una mayor aceptación. En las Figuras 1 y 2 se presenta el path diagram del modelo de cuatro dimensiones tanto en PSP como en PSN respectivamente, con sus correspondientes pesos estandarizados, cuyos valores oscilaron en los PSP entre .25 del ítem 12 y .86 del ítem 15, y las correlaciones entre las dimensiones obtuvieron valores de entre .48 y .85. Para los
PSN los valores de los pesos estandarizados oscilaron entre .02 del ítem 52 y .87 del ítem 10 y las correlaciones entre factores fueron de entre .28 y .89 .

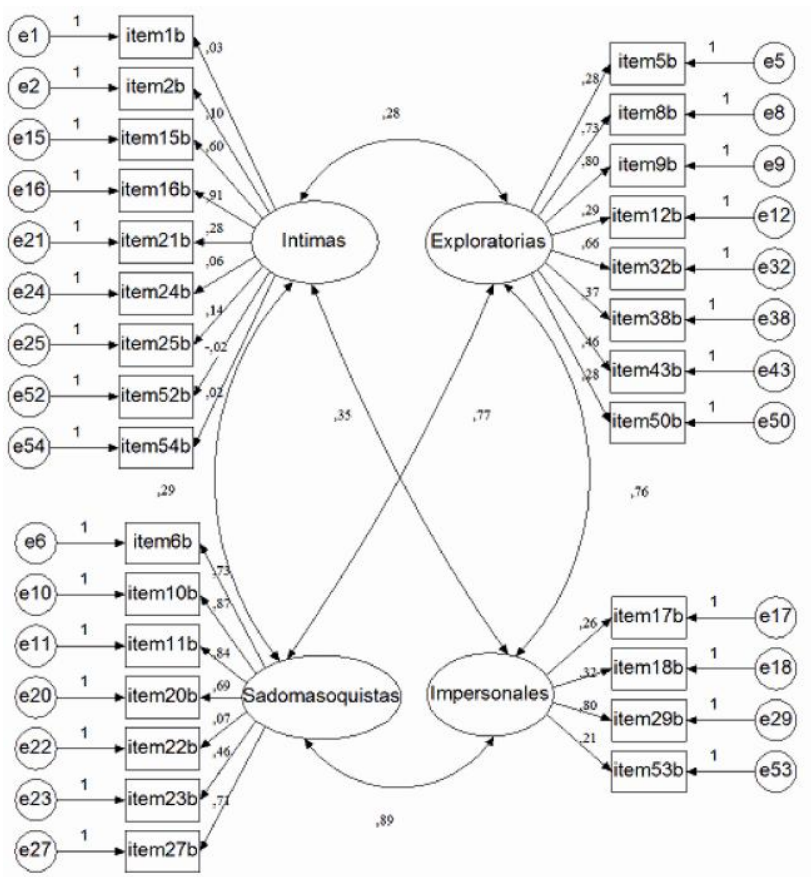

Figura 2. Path diagram del modelo de cuatro factores correspondiente a PSN del SCC-28.

\section{Análisis de invarianza}

Una vez confirmada la estructura factorial de cuatro dimensiones en los PSP y PSN, se procedió a evaluar la invarianza del modelo por sexo en ambos PSP y PSN. Para ello, se realizó la evaluación progresiva o secuencial de la invarianza configural (igualdad en el modelo sin restricciones), invarianza métrica (igualdad en la saturación de los pesos o cargas factoriales, invarianza fuerte (igualdad entre las varianzas y covarianzas) e invarianza estricta (igualdad en la varianza de errores), mediante modelos de ecuaciones estructurales (véase Byrne, 2008; Elosúa, 2005). Se consideró indicio de invarianza que el CFI no disminuyese más de .01 con respecto al modelo anterior (Cheung y Rensvold, 2002).

$\mathrm{El}$ análisis de invarianza entre hombres y mujeres en los PSP mostró la existencia de invarianza configural, al considerarse los valores RMSEA y GFI aceptables, pero no existió invarianza métrica, dado que CFI disminuyó más de .01 
con respecto al modelo anterior. Existe por tanto cierta estabilidad en los modelos de medida entre hombres y mujeres, demostrándose la equivalencia del constructo, aunque la invarianza alcanzada es sólo débil. Al analizar la invarianza por sexo en los PSN, existe invarianza estricta, lo cual permite realizar comparaciones libres de sesgos entre hombres y mujeres en el modelo, al alcanzar el mayor nivel de acuerdo entre estructuras factoriales (véase la Tabla 4).

Tabla 4. Índices de bondad de ajuste de los modelos de invarianza.

\begin{tabular}{|c|c|c|c|c|c|}
\hline & $\chi^{2}$ & $\mathrm{gl}$ & RMSEA & GFI & CFI \\
\hline \multicolumn{6}{|c|}{ Diferencias entre hombres y mujeres en PSP } \\
\hline Invarianza configural & 2525.19 & 690 & .04 & .86 & .34 \\
\hline Invarianza métrica & 2648.90 & 717 & .04 & .85 & .30 \\
\hline Invarianza fuerte & 2690.27 & 727 & .04 & .85 & .29 \\
\hline Invarianza estricta & 2970.75 & 751 & .04 & .84 & .20 \\
\hline \multicolumn{6}{|c|}{ Diferencias entre hombres y mujeres en PSN } \\
\hline Invarianza configural & 5277.00 & 690 & .05 & .85 & .25 \\
\hline Invarianza métrica & 5278.21 & 717 & .04 & .85 & .25 \\
\hline Invarianza fuerte & 5286.33 & 727 & .04 & .85 & .25 \\
\hline Invarianza estricta & 5286.34 & 751 & .04 & .85 & .25 \\
\hline
\end{tabular}

\section{Evidencias de validez}

Como evidencias de validez, se calcularon las correlaciones entre las dimensiones que configuran PSP y PSN. Tal como se muestra en la Tabla 5, todas las dimensiones de PSP y PSN correlacionaron entre sí, obteniéndose las correlaciones más elevadas entre Fantasías Exploratorias e Impersonales y las más bajas entre Fantasías Sadomasoquistas e Intimas de modo similar para PSP y PSN.
Tabla 5. Correlaciones bivariadas entre las dimensiones de PSP y PSN del SCC.

\begin{tabular}{lllll}
\hline & $F 1$ & $F 2$ & $F 3$ & $F 4$ \\
\hline F1. Fantasias Intimas & - & $.52^{* *}$ & $.27^{* *}$ & $.59^{* *}$ \\
F2. Fantasias Exploratorias & $.53^{* *}$ & - & $.66^{* *}$ & $.69^{* *}$ \\
F3. Fantasias Sadomasoquistas & $.39^{* *}$ & $.61^{* *}$ & - & $.63^{* *}$ \\
F4. Fantasias Impersonales & $.52^{* *}$ & $.65^{* *}$ & $.53^{* *}$ & -
\end{tabular}

Nota. ${ }^{* *} p<.01$. Parte inferior izquierda relativa a PSP; parte superior derecha relativa a PSN.

Asimismo, se examinaron las relaciones de las dimensiones de PSP y PSN con variables afines. Al analizar la relación entre PSP y actitud favorable hacia las fantasías y ensoñación sexual, se realizaron correlaciones parciales, controlando el efecto de los PSN, al demostrarse una correlación significativa entre ambos tipos de pensamiento $(r=.31 p=$ .000), y del sexo, al no haberse alcanzado invarianza métrica entre hombres y mujeres. Para el análisis de los PSN se controló sólo el efecto de los PSP. La actitud favorable hacia las fantasías sexuales correlacionó de forma positiva con todas las dimensiones de PSP, oscilando los coeficientes entre .30 (Fantasias Sadomasoquistas) y .50 (Fantasías Íntimas). Por su parte, las tres dimensiones de ensoñación sexual presentaron también correlaciones positivas con las dimensiones de PSP, dándose la correlación más baja entre intensidad de ensoñaciones sexuales y Fantasías Sadomasoquistas, y la más elevada entre tendencia a ensoñaciones heterosexuales y Fantasias Exploratorias. Por lo que respecta a PSN, se encontró que tener una actitud favorable hacia las fantasías correlacionó en sentido negativo con todas las dimensiones de PSN. Asimismo, la tendencia a ensoñaciones heterosexuales mostró una correlación negativa con todas las dimensiones y la intensidad de las ensoñaciones sexuales mostró una correlación negativa con Fantasías Sadomasoquistas, aunque en general las correlaciones obtenidas en los PSN fueron muy bajas (véase la Tabla 6).

Tabla 6. Correlaciones parciales entre las dimensiones de PSP y PSN del SCC y variables criterio.

\begin{tabular}{|c|c|c|c|c|c|c|c|c|}
\hline & \multicolumn{4}{|c|}{ Pensamientos Sexuales Positivos } & \multicolumn{4}{|c|}{ Pensamientos Sexuales Negativos } \\
\hline & F1 & F2 & F3 & F4 & F1 & F2 & F3 & F4 \\
\hline Actitud favorable fantasías sexuales & $.50 * * *$ & $.42^{* * *}$ & $.30 * * *$ & $.47 * * *$ & $-.08^{* *}$ & $-.11 * * *$ & $-.12 * * *$ & $-.12 * * *$ \\
\hline Tendencia ensoñaciones heterosexuales & $.32^{* * *}$ & $.46^{* * *}$ & $.38 * * *$ & $.40^{* * *}$ & -.01 & $-.07 * *$ & $-.08 * * *$ & $-.06^{*}$ \\
\hline Intensidad ensoñaciones sexuales & $.37 * * *$ & $.34 * * *$ & $.26 * * *$ & $.32 * * *$ & -.00 & -.03 & $-.05^{*}$ & -.04 \\
\hline Ensoñaciones eróticas personalizadas & $.28 * * *$ & $.39 * * *$ & $.30 * * *$ & $.33^{* * *}$ & .03 & .00 & -.04 & -.00 \\
\hline
\end{tabular}

\section{Discusión}

Debido a una nueva visión más completa del constructo y dada la ausencia de instrumentos en castellano que permitan evaluar, no solo la frecuencia, sino también el modo en que se experimentan las fantasías sexuales, el objetivo de este estudio fue adaptar y validar al castellano el Sexual Cognitions Checklist de Renaud y Byers (1999).

A través del análisis de contenido se configuró la estructura factorial del instrumento, de acuerdo a las dimensiones teóricas propuestas por Wilson (1988): fantasías sexuales íntimas, exploratorias, sadomasoquistas e impersonales recordemos que es el instrumento en el que se fundamenta el SCC-. Tras el juicio de expertos realizado, la versión inicial de 56 ítems, quedó reducida a una de 28. La utilización de esta técnica como primer filtro para la depuración del instrumento ha sido un requisito indispensable, considerando entre otros motivos la ausencia de una previa validación del SCC que pudiera guiar el presente estudio y la dificultad que han mostrado anteriores estudios psicométricos en pobla- 
ción española de establecer la estructura dimensional del WSFQ (Wilson, 1988) a través de pruebas exploratorias. En concreto, el estudio realizado por Carvajal et al. (1990) obtiene, a través de un análisis exploratorio, hasta seis dimensiones, difiriendo algunos de los ítems en su agrupación con respecto al planteamiento original de Wilson (1988). Asimismo, Sierra, Ortega, Martín-Ortiz y Vera-Villaroel (2004) realizaron una validación del instrumento, siendo dicha versión reducida a 24 ítems por conseguir un mejor ajuste al someterse a un análisis factorial confirmatorio (Sierra et al., 2006). Al comparar estas investigaciones previas con el presente estudio, se observa la solidez de 12 de los 40 ítems que componen la versión original del WSFQ (Wilson, 1988), en concreto los ítems 2, 8, 9, 11, 12, 16, 17, 25, 32, 50, 52 y 53. Por otro lado, son siete los ítems que no consiguen mantenerse en ninguno de los estudios realizados en población española (ítems 30, 31, 33, 40, 44, 48 y 49), siendo todos ellos calificados por los expertos en el presente estudio como no pertinentes con el constructo de pensamientos sexuales, a excepción de los ítems 40 y 44, que aun consiguiendo dicho acuerdo, no lograron ubicarse en alguna de las dimensiones propuestas. Esta falta de consistencia en la obtención de una estructura coincidente con la propuesta teórica de Wilson (1988) podría deberse, además de a las posibles diferencias culturales, a otros aspectos relativos al WSFQ (Wilson, 1988), como el uso de una terminología vaga (O'Donohue, Letourneau y Dowling, 1997) y a la generalidad en las conductas sexuales y contenido de sus ítems (Baumgartner, Scalora y Huss, 2002).

Al observar las medias alcanzadas por cada dimensión, se evidencia que las fantasías que se experimentan con mayor frecuencia de modo positivo son las íntimas, seguidas de las exploratorias, impersonales y sadomasoquistas, de modo similar a estudios previos (Wilson y Lang, 1981; Zubeidat, Ortega, del Villar y Sierra, 2003). En concreto, la fantasía más frecuentemente experimentada es Practicar un coito con una pareja amada y la menos frecuente, de contenido sadomasoquista es Ser una víctima sexual. Por otro lado, los PSN más frecuentemente experimentados son de tipo sadomasoquista, seguidos de las fantasías exploratorias, impersonales e íntimas. En particular, el pensamiento que resulta más negativo es Ser presionado/ a a mantener relaciones sexuales, mientras que el experimentado como menos negativo es Ser excitado por material o ropa. Se observa, por un lado, que los resultados son coincidentes con los estudios de Renaud y Byers (1999, 2001), en los que las fantasías positivas más frecuentes son aquellas de contenido romántico e íntimo, y las fantasías negativas más destacables están asociadas a la dominación y sumisión, características de la dimensión sadomasoquista. Asimismo, pensamientos sexuales positivos y negativos están significativamente relacionados, demostrándose el solapamiento entre ambos tipos de fantasía, tal como indicaron Renaud y Byers (2001). A través de estos hallazgos, se destaca, por tanto, la simultaneidad y no exclusividad de ambos tipos de fantasía (Renaud y Byers, 2001), al poder experimentarse un mismo pensamiento de forma positiva y negativa, siendo el contenido de las mismas insuficiente para determinar si el pensamiento es positivo o negativo (Renaud y Byers, 1999). En definitiva, un pensamiento puede resultar muy positivo y no estar por ello asociado a una menor negatividad.

Respecto a la fiabilidad de la escala, la dimensión que alcanza una mayor consistencia es Fantasías Intimas y la que presenta el valor más bajo es Impersonales. Es necesario señalar que la fiabilidad alcanzada en cada dimensión es similar entre PSP y PSN, destacando la consistencia entre ambos tipos de fantasía sexual. Los valores obtenidos son comparables a los encontrados por Sierra et al. (2004), al informar de una mayor fiabilidad para la subescala de Fantasías Intimas, con un alfa de .79 y menor homogeneidad en la subescala Impersonales, igual a .66, replicándose valores similares en un estudio posterior (Sierra et al., 2006). Resulta de interés considerar que las Fantasias Impersonales suelen presentar en gran parte de los estudios, valores más bajos de consistencia, lo que hace cuestionarnos si el contenido de esta dimensión no es suficientemente sólido o si podría estar solapado con el de otras dimensiones. En este sentido, no es posible establecer comparaciones con la versión original del WSFQ (Wilson, 1988) ya que no se ofrece información sobre la fiabilidad de la escala, siendo esta una limitación considerable, al no ofrecer una prueba empírica que contribuya a la consolidación de sus dimensiones.

A pesar de las comentadas limitaciones del instrumento en el que se fundamenta el SCC (Renaud y Byers, 1999; Renaud y Byers, 2011), la estructura tetrafactorial ofrece, a través de un análisis factorial confirmatorio, y cuando se compara con un modelo unidimensional, un mejor ajuste para PSP y PSN. Debe considerarse que los valores de ajuste alcanzados en el modelo tetrafactorial son aceptables, aunque rozan el límite de aceptabilidad, especialmente al poner a prueba el modelo PSN, para el que uno de los índices no alcanza un nivel aceptable. Ante los resultados obtenidos, se hace necesario abordar por tanto algunas cuestiones importantes: 1) la construcción original del instrumento, basada en la combinación de ítems de dos cuestionarios independientes podría no representar de forma sólida la dimensionalidad de fantasías sexuales propuesta por Wilson (1988) y 2) los pensamientos sexuales negativos podrían ser diferentes en contenido a las fantasías sexuales propuestas a través del SCC, por lo que la esencia de este tipo diferente de fantasías podría no estar claramente reflejada en el SCC.

Posteriormente, y con el objetivo de asegurar la equivalencia de ambos modelos entre hombres y mujeres, se ha realizado un análisis progresivo de invarianza. Los resultados permiten indicar, por un lado, que el modelo tetrafactorial en los PSP resulta equivalente para hombres y mujeres solo a un nivel configural, existiendo desajuste a partir de los pesos o cargas factoriales. Estas limitaciones no están presentes al realizar comparaciones por sexo en los PSN, dado que al alcanzar invarianza estricta, se asume la plena equivalencia en el constructo. En este sentido, sería necesario esclarecer las causas de desajuste entre los modelos por sexo en los PSP. 
En cuanto a los indicadores de validez, se observa por un lado que todas las dimensiones tanto en PSP como en PSN correlacionan entre sí, produciéndose en ambos casos, una mayor relación entre fantasías impersonales y exploratorias, y menor entre íntimas y sadomasoquistas. En cuanto a la asociación de PSP y PSN con otros constructos afines, se encuentra que la actitud favorable hacia las fantasías está relacionada con tener una mayor frecuencia de pensamientos sexuales positivos; en concreto, la actitud favorable obtiene mayor relación con fantasías íntimas y menor con fantasías sadomasoquistas. Los resultados son similares a los señalados por Desvarieux et al. (2005). Asimismo, tener una actitud favorable hacia las fantasías está inversamente relacionado con experimentar pensamientos sexuales negativos. De este modo, la actitud resulta una variable de gran importancia que se haya vinculada al modo en que se experimentan las fantasías sexuales, tal como se había evidenciado en otros estudios (Sierra, Perla y Gutiérrez-Quintanilla, 2010; Sierra et al., 2003; Zubeidat et al., 2004), destacándose además lo apuntado por Desvarieux et al. (2005) sobre la importancia de considerar en el marco terapéutico las actitudes del paciente hacia las fantasías para considerar posteriormente el contenido de las mismas. Algunos estudios apuntan al posible carácter disfuncional de las fantasías sadomasoquistas (Sierra et al., 2006), al demostrarse su relación con insatisfacción sexual (Wilson, 1988) y su interferencia con el deseo (Zubeidat et al., 2003).

Por otro lado, se verifica que la imaginación es un elemento fundamental de la fantasía sexual (Leitenberg y Henning, 1995; Pérez-González, Moyano y Sierra, 2011), al encontrarse relación entre la capacidad de ensoñación sexual y todas las dimensiones de PSP. Asimismo, la dimensión tendencia de ensoñaciones heterosexuales mostró una relación negativa con todos los PSN. Los resultados van en la línea de los encontrados por Viedma et al. (2005), quienes demuestran que, de modo similar al presente estudio, la tendencia de ensoñaciones heterosexuales está más fuertemente asociada con las fantasías exploratorias, en tanto que la in-

\section{Referencias}

Baumgartner, J.V., Scalora, M.J. y Huss, T. (2002). Assessment of the Wilson Sex Fantasy Questionnaire among child molesters and nonsexual forensic offenders. Sexual Abuse: A Journal of Research and Treatment, 9, 19-30.

Bentler, P.M. (1989). EQS structural equations program manual. Los Angeles: BMDP. Biddle, S.J.H.

Browne, M.W. y Cudeck, R. (1993). Alternative ways of assessing model fit. En K. Bollen y J. Long (Eds.), Testing structural equation models, (pp. 136162). Londres: Sage.

Byers, E.S., Purdon, C. y Clark, D.A. (1998). Sexual intrusive thoughts of college students. Journal of Sex Research, 35, 359-369.

Byrne, B. (2008). Testing for multigroup equivalence of a measuring instrument: A walk through the process. Psicothema, 20, 872-882.

Carretero-Dios, H. y Pérez, C. (2007). Standards for the development and review of instrumental studies: Considerations about test selection in psychological research. International Journal of Clinical and Health Psychology, 7, 863-882. tensidad de ensoñaciones sexuales lo está con fantasías íntimas. Algunos pensamientos sexuales negativos muestran relación con tendencia de ensoñaciones heterosexuales al presentar correlaciones negativas significativas, aunque muy bajas, con fantasías exploratorias, sadomasoquistas e impersonales y entre intensidad de ensoñaciones sexuales y fantasías sadomasoquistas. Esta relación negativa, viene dada por la propia naturaleza de los pensamientos sexuales negativos, al estar caracterizados por una mayor intrusividad, que resulta en mayores intentos por controlarlos y presentar una menor duración que los pensamientos sexuales positivos (Renaud y Byers, 1999).

En definitiva, este estudio sobre la adaptación y validación del Sexual Cognitions Checklist en población española, además de dar apoyo a la multidimensionalidad del constructo, ofrece un instrumento con apropiadas garantías psicométricas que permite examinar de modo distinguible las fantasías sexuales como pensamientos sexuales positivos y negativos. Esta distinción se hace especialmente relevante al considerar que una perspectiva positiva de la sexualidad humana es un aspecto fundamental para la capacidad de disfrutar y expresar la sexualidad (Lottes, 2000), siendo la expresión de la sexualidad un derecho que hay que garantizar para cualquier persona en un marco satisfactorio y saludable (Lameiras-Fernández et al., 2010; Santos-Iglesias y Sierra, 2010). Por todo ello, futuros estudios deberán analizar el papel de las fantasías sexuales negativas y examinar su posible carácter disfuncional sobre la salud sexual.

Agradecimientos.- Este trabajo ha sido apoyado y financiado por el Ministerio de Educación a través de la Beca Predoctoral FPU (Referencia: AP2008-02503). A Hugo Carretero-Dios por su asesoramiento, a María Pilar Sánchez López, por brindar su apoyo y colaboración durante la estancia realizada en la Universidad Complutense de Madrid y a todos los Centros colaboradores en el estudio: Facultad de Psicología de la Universidad Complutense de Madrid, Biblioteca de Andalucía en Granada, Centros de Educación Permanente de Adultos, Ciclos Formativos y Talleres de empleo en Andalucía.

Carvajal, F., Sebastián, J., Cornide, E., Delgado, A., Castellote, I. y Blanco, C. (1990). Implicaciones del modelo de la androginia en el comportamiento sexual: Arousal subjetivo y fantasías sexuales. Revista de Psicología Social, 5, 23-42.

Chau, Y.K. (1997). Re-examining a model for evaluating information center success using a structural equation modelling approach. Decision Sciences, 28, 309-334.

Cheung, G.W. y Rensvold, R.B. (2002). Evaluating goodness-of-fit indexes for testing measurement invariance. Structural Equation Modeling, 9, 235255.

Davis, L. (1992). Instrument review: Getting the most from your panel of experts. Applied Nursing Research, 5, 104-107.

Desvarieux, A.R., Salamanca, Y., Ortega, V. y Sierra, J.C. (2005). Validación de la versión en castellano del Hurlbert Index of Sexual Fantasy: Una medida de actitud hacia las fantasías sexuales. Revista Mexicana de Psicologia, 22, 529-539. 
Elosúa, P. (2005). Evaluación progresiva de la invarianza factorial entre las versiones original y adaptada de una escala de autoconcepto. Psicothema, 17, 356-362.

Fuertes, A. y López, F. (1997). Aproximaciones al estudio de la sexualidad. Salamanca: Amarú.

Giambra, L.M. y Singer, J.L. (1998). Sexual Daydreaming Scale of the Imaginal Process Inventory. En C.M. Davis, W.L. Yarber, R. Bauserman, G. Schreer y S.L. Davis (Eds.), Handbook of sexuality- related measures (pp. 234-235). Londres: Sage.

Hambleton, R.K. (1993). Translating achievement test for use in crossnational studies. European Journal of Psychological Assessment, 9, 57-68.

Hambleton, R.K. (1996). Adaptación de tests para su uso en diferentes idiomas y culturas: fuentes de error, posibles soluciones y directrices prácticas. En J. Muñiz (Ed.), Psicometría (pp. 207-238). Madrid: Universitas, S.A.

Hambleton, R.K. y Patsula, L. (1999). Increasing the validity of Adapted Tests: Myths to be avoided and guidelines for improving test adaptation practices. Journal of Applied Testing Technology, 1, 1-16.

Hartwick, J. y Barki, H. (1994). Explaining the role of user participation in information system use. Management Science, 40, 440-465.

Hu, L. y Bentler, P.M. (1999). Cutoff criteria for fit indexes in covariance structure analysis: Conventional criteria versus new alternatives. Structural Equation Modeling: A multidisciplinary journal, 6, 1-55.

Hurlbert, D.F. y Apt, C. (1993). Female sexuality: A comparative study between women in homosexual and heterosexual relationships. Journal of Sex and Marital Therapy, 19, 315-327.

Jöreskog, K.G. y Sörbom, D. (1993). LISREL8: Structural equation modeling with the SIMPLIS command language. Hillsdale, NJ: Erlbaum.

Lameiras-Fernández, M., Carrera-Fernández, M.V., Faílde-Garrido, J.M., Ricoy-Lorenzo, M.C., López-Castedo, A. y Núñez-Mangana, A.M. (2010). Promocionando el uso del preservativo femenino: un estudio cualitativo en parejas heterosexuales españolas. International Journal of Clinical and Health Psychology, 10, 309-326.

Leitenberg, H. y Henning, K. (1995). Sexual fantasy. Psychological Bulletin, 117, $469-496$

Lottes, I. (2000). New perspectives on sexual health. En I. Lottes y O. Kontula (Eds.), New views on sexual health: The case of Finland (pp. 7-29). Helsinki, Finland: Population Research Institute.

Montero, I. y León, O.G. (2007). A guide for naming research studies in Psychology. International Journal of Clinical and Health Psychology, 7, 847862.

Nunnally, J.C. y Bernstein, I.J. (1995). Teoría psicométrica. Madrid: McGrawHill.

Nutter, D.E. y Condron, M.K. (1985). Sexual fantasy and activity patterns of males with inhibited sexual desire and males with erectile dysfunction versus normal controls. Journal of Sex and Marital Therapy, 11, 91-98.

O'Donohue, W., Letourneau, E.J. y Dowling, H. (1997). Development and preliminary validation of a paraphilic sexual fantasy questionnaire. Sexual abuse: A Journal of Research and Treatment, 9, 167-178.

Pérez-González, S., Moyano, N. y Sierra, J.C. (2011). La capacidad de ensoñación sexual: su relación con la actitud hacia las fantasías sexuales y rasgos de personalidad. Cuadernos de Medicina Psicosomática y Psiquiatría de Enlace, 99, 9-20.

Perla, F., Sierra, J.C., Vallejo-Medina, P. y Gutiérrez-Quintanilla, R. (2009). Un estudio psicométrico de la versión española reducida del Hurlbert Index of Sexual Fantasy. Boletin de Psicologia, 96, 7-16.

Purdon, C. y Clark, D.A. (1994). Perceived control and appraisal of obsessional intrusive thoughts. A replication and extension. Behavioral and Cognitive Psychotherapy, 22, 269-286.

Purifoy, F.E., Grodsky, A. y Giambra, L.M. (1992). The relationship of sexual daydreaming to sexual activity, sexual drive, and sexual attitudes for women across the life-span. Archives of Sexual Behavior, 21, 369-385.
Renaud, C.A. y Byers, E.S. (1999). Exploring the frequency, diversity, and content of university students' positive and negative sexual cognitions. Canadian Journal of Human Sexuality, 8, 17-30.

Renaud, C.A. y Byers, E.S. (2001). Positive and negative sexual cognitions: Subjective experience and relationships to sexual adjustment. Journal of Sex Research, 38, 252-262.

Renaud, C.A. y Byers, E.S. (2011). Sexual Cognitions Checklist. En T.D. Fisher, C.M. Davis, W.L. Yarber, R. Bauserman, G. Scherer y S.L. Davis (Eds.), Handbook of sexuality-related measures (pp. 110-112). Nueva York: Routledge.

Santos-Iglesias, P., Calvillo, G. y Sierra, J.C. (2011). A further examination of Levine's model of sexual desire. Psychology \& Sexuality. Avance publicación electrónica. doi:10.1080/19419899.2011.576697

Santos-Iglesias, P. y Sierra, J.C. (2010). El papel de la asertividad sexual en la sexualidad humana: una revisión sistemática. International Journal of Clinical and Health Psychology, 10, 553-577.

Schumacker, R.E. y Lomax, R.G. (2004). A beginner's guide to structural equation modeling. Mahwah, NJ: Lawrence Erlbaum Associates.

Selby-Harrington, M.L., Mehta, S.M., Jutsum, V., Riportella-Muller, R. y Quade, D. (1994). Reporting of $i$

nstrument validity and reliability in selected clinical nursing journals, 1989. Journal of Professional Nursing, 10, 47-56.

Sierra, J.C. y Buela-Casal, G. (2002). Evaluación y tratamiento de las disfunciones sexuales. En G. Buela-Casal y J.C. Sierra (Eds.), Manual de evaluación y tratamientos psicológicos (pp. 439-485). Madrid: Biblioteca Nueva.

Sierra, J.C., Ortega, V., Martín-Ortiz, J.D. y Vera-Villaroel, P. (2004). Propiedades psicométricas del Cuestionario de Wilson de Fantasías Sexuales. Revista Mexicana de Psicología, 21, 37-50.

Sierra, J.C., Ortega, V. y Zubeidat, I. (2006). Confirmatory factor analysis of a Spanish version of the Sex Fantasy Questionnaire: Assessing gender differences. Journal of Sex and Marital Therapy, 32, 137-159.

Sierra, J.C., Perla, F. y Gutiérrez-Quintanilla, R. (2010). Actitud hacia la masturbación en adolescentes: propiedades psicométricas de la versión española del Attitudes Toward Masturbation Inventory. Universitas Psychologica, 9, 531-542.

Sierra, J.C., Zubeidat, I., Carretero-Dios, H. y Reina, S. (2003). Estudio psicométrico preliminar del Test de Deseo Sexual Inhibido en una muestra española no clínica. International Journal of Clinical and Health Psychology, 3, 489-504.

Trudel, G. (2002). Sexuality and marital life: Results of a survey. Journal of Sex and Marital Therapy, 28, 229-249.

Viedma, I., Gutiérrez, P., Ortega, V. y Sierra, J.C. (2005). Escala de ensoñaciones sexuales: Primeros datos psicométricos en muestras españolas. Análisis y Modificación de Conducta, 31, 406-431.

Wilson, G.D. (1988). Measurement of sex fantasy. Sexual and Marital Therapy, 3, 45-55.

Wilson, G.D. y Lang, R.J. (1981). Sex differences in sexual fantasy patterns. Personality and Individual Differences, 2, 343-346.

Zamboni, B.D. y Crawford, I. (2002). Using masturbation in sex therapy: Relationships between masturbation, sexual desire, and sexual fantasy. Journal of Psychology and Human Sexuality, 14, 123-141.

Zimmer, D., Borchardt, E. y Fischle, C. (1983). Sexual fantasies of sexually distressed and non-distressed men and women: An empirical comparison. Journal of Sex and Marital Therapy, 9, 38-50.

Zubeidat, I., Ortega, V. y Sierra, J.C. (2004). Evaluación de algunos factores determinantes del deseo sexual: Estado emocional, actitudes sexuales y fantasías sexuales. Análisis y Modificación de Conducta, 30, 105-128.

Zubeidat, I., Ortega, V., del Villar, C. y Sierra, J.C. (2003). Un estudio sobre la implicación de las actitudes y fantasías sexuales en el deseo sexual de los adolescentes. Cuadernos de Medicina Psicosomática y Psiquiatría de Enlace, $67 / 68,71-78$.

(Artículo recibido: 27-4-2011, revisado: 5-11-2011, aceptado: 12-11-2011 
Apéndice 1. Versión española del Sexual Cognitions Checklist de Renaud y Byers (1999).

A continuación encontrará una serie de pensamientos sexuales. En cada uno de ellos, usted debe indicar la frecuencia con la que los ha tenido en forma de pensamiento sexual positivo (pensamientos aceptables, agradables y placenteros, que surgen durante la masturbación, al mantener una relación sexual y/o mientras se realizan actividades no sexuales) y de pensamiento sexual negativo (pensamientos inaceptables, desagradables y no placenteros, que surgen durante la masturbación, al mantener una relación sexual y/o mientras se realizan actividades no sexuales).

$0=\underline{\text { Nunca }}$ he tenido este pensamiento

$1=$ He tenido este pensamiento una o dos veces en mi vida

$2=$ He tenido este pensamiento algunas veces al año

$3=$ He tenido este pensamiento una o dos veces al mes

$4=$ He tenido este pensamiento una o dos veces a la semana

$5=$ He tenido este pensamiento diariamente

$6=$ He tenido (tengo) este pensamiento de forma frecuente durante el día

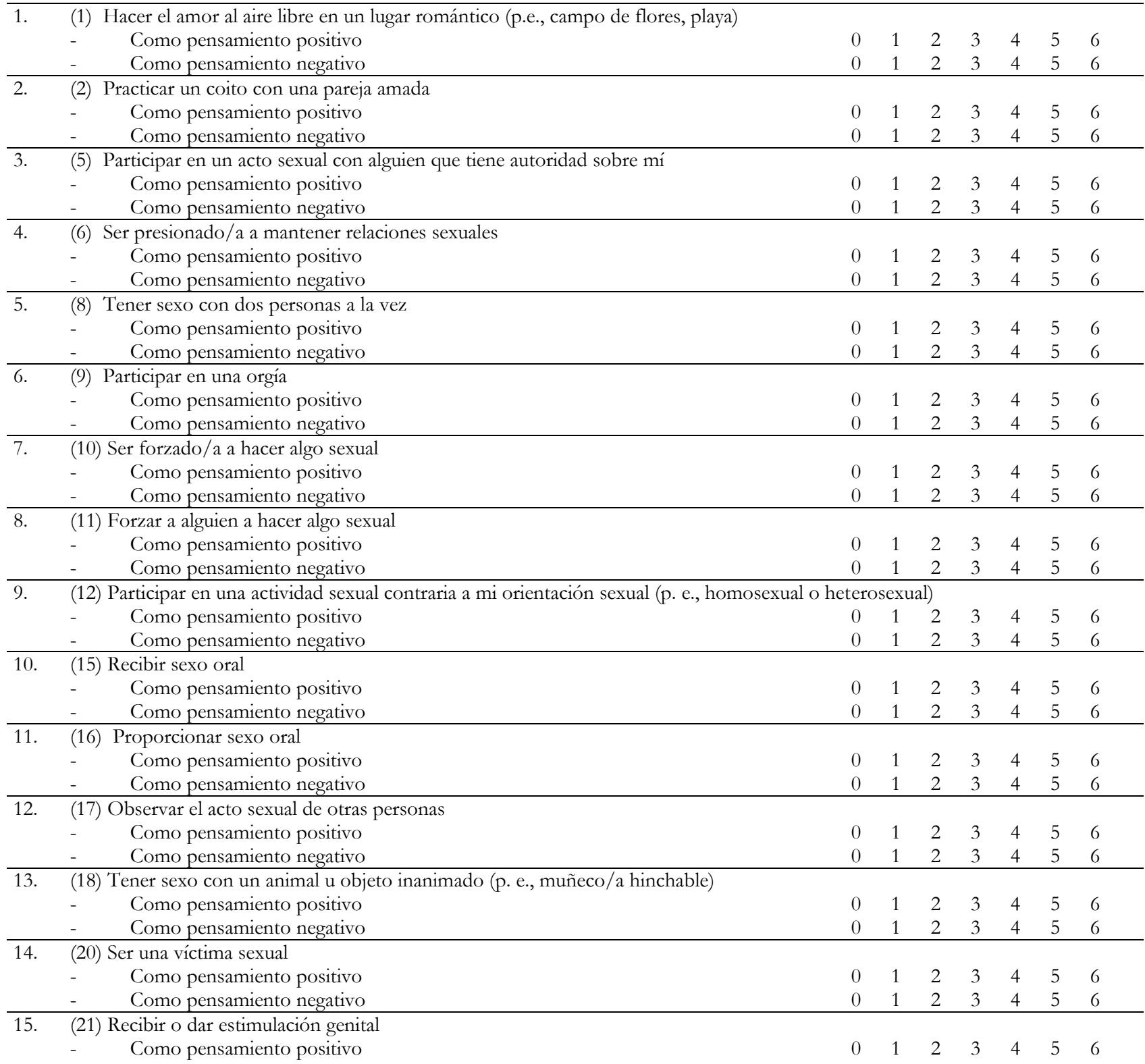


Como pensamiento negativo $\begin{array}{lllllll}0 & 1 & 2 & 3 & 4 & 5 & 6\end{array}$

\begin{tabular}{clc}
\hline 16. & (22) Azotar o golpear en el trasero a alguien \\
& - & Como pensamiento positivo \\
& - & Como pensamiento negativo \\
\hline 17. & (23) Ser azotado/a o golpeado/a en el trasero \\
& $-\quad$ Como pensamiento positivo \\
& $-\quad$ Como pensamiento negativo \\
\hline
\end{tabular}

18

(24) Desnudar a alguien

Como pensamiento positivo $\quad \begin{array}{lllllllll}0 & 0 & 1 & 2 & 3 & 4 & 5 & 6\end{array}$

Como pensamiento negativo

$\begin{array}{lllllll}0 & 1 & 2 & 3 & 4 & 5 & 6\end{array}$

$\begin{array}{lllllll}0 & 1 & 2 & 3 & 4 & 5 & 6\end{array}$

19. (25) Ser desnudado/a
$\quad-\quad$ Como pensamiento positivo

Como pensamiento negativo

$\begin{array}{lllllll}0 & 1 & 2 & 3 & 4 & 5 & 6\end{array}$

$\begin{array}{lllllll}0 & 1 & 2 & 3 & 4 & 5 & 6\end{array}$

orzar a otra persona a mantener relaciones sexuales conmigo

Como pensamiento positivo

- Como pensamiento negativo

$\begin{array}{lllllll}0 & 1 & 2 & 3 & 4 & 5 & 6\end{array}$

$\begin{array}{lllllll}0 & 1 & 2 & 3 & 4 & 5 & 6\end{array}$

Como pensamiento positivo

$\begin{array}{lllllll}0 & 1 & 2 & 3 & 4 & 5 & 6\end{array}$

21. (29) Ser excitado/a por material o ropa (p.e., látex, cuero, ropa interior)

Como pensamiento negativo

$\begin{array}{lllllll}0 & 1 & 2 & 3 & 4 & 5 & 6 \\ 0 & 1 & 2 & 3 & 4 & 5 & 6\end{array}$

$\begin{array}{lllllll}0 & 1 & 2 & 3 & 4 & 5 & 6\end{array}$

\begin{tabular}{|c|c|c|c|c|c|c|c|c|}
\hline & Como pensamiento negativo & 0 & 1 & 2 & 3 & 4 & 5 & 6 \\
\hline \multirow[t]{3}{*}{22.} & (32) Intercambio de parejas & & & & & & & \\
\hline & - Como pensamiento positivo & 0 & 1 & 2 & 3 & 4 & 5 & 6 \\
\hline & Como pensamiento negativo & 0 & 1 & 2 & 3 & 4 & 5 & 6 \\
\hline \multirow[t]{3}{*}{23.} & (38) Tener sexo en un lugar público & & & & & & & \\
\hline & - Como pensamiento positivo & 0 & 1 & 2 & 3 & 4 & 5 & 6 \\
\hline & Como pensamiento negativo & 0 & 1 & 2 & 3 & 4 & 5 & 6 \\
\hline \multirow[t]{3}{*}{24.} & (43) Ser promiscuo/a & & & & & & & \\
\hline & Como pensamiento positivo & 0 & 1 & 2 & 3 & 4 & 5 & 6 \\
\hline & Como pensamiento negativo & 0 & 1 & 2 & 3 & 4 & 5 & 6 \\
\hline \multirow[t]{3}{*}{25.} & (50) Tener sexo con alguien de diferente raza & & & & & & & \\
\hline & - Como pensamiento positivo & 0 & 1 & 2 & 3 & 4 & 5 & 6 \\
\hline & - $\quad$ Como pensamiento negativo & 0 & 1 & 2 & 3 & 4 & 5 & 6 \\
\hline \multirow[t]{3}{*}{26.} & (52) Ser masturbado/a hasta el orgasmo por la pareja & & & & & & & \\
\hline & - $\quad$ Como pensamiento positivo & 0 & 1 & 2 & 3 & 4 & 5 & 6 \\
\hline & Como pensamiento negativo & 0 & 1 & 2 & 3 & 4 & 5 & 6 \\
\hline \multirow[t]{3}{*}{27.} & (53) Contemplar imágenes o películas obscenas & & & & & & & \\
\hline & Como pensamiento positivo & 0 & 1 & 2 & 3 & 4 & 5 & 6 \\
\hline & - $\quad$ Como pensamiento negativo & 0 & 1 & 2 & 3 & 4 & 5 & 6 \\
\hline \multirow[t]{3}{*}{28.} & (54) Besar apasionadamente & & & & & & & \\
\hline & - $\quad$ Como pensamiento positivo & 0 & 1 & 2 & 3 & 4 & 5 & 6 \\
\hline & Como pensamiento negativo & 0 & 1 & 2 & 3 & 4 & 5 & 6 \\
\hline
\end{tabular}

Nota.- Entre paréntesis aparece el número correspondiente a la versión inicial de 56 ítems y a la que se hace referencia a lo largo del artículo. 